\title{
Fluticasone/salmeterol is associated with a higher risk of pneumonia and pneumonia related events in comparison with budesonide/formoterol in patients with COPD
}

Combination treatment with inhaled corticosteroids and long acting $\beta_{2}$ agonists decrease exacerbations and improve quality of life in patients with chronic obstructive pulmonary disease (COPD) however; a potential concern is an increased risk of pneumonia. Although previous studies have shown the risk of pneumonia to increase with fluticasone/salmeterol versus placebo and tiotropium, there has been no direct comparison of fixed combinations of inhaled corticosteroid/long acting $\beta_{2}$ agonists.

The PATHOS study compared the occurrence of pneumonia and pneumonia related events in patients with COPD treated with either fluticasone/salmeterol (Seretide) or budesonide/formoterol (Symbicort). This was an observational retrospective cohort study matched for propensity score comparing 2734 patients in each group. During the study period 2115 patients (39\%) had at least one diagnosis of pneumonia. Compared with the budesonide/formoterol group, the rate of pneumonia and rate of admission to hospital related to pneumonia were $73 \%$ and $74 \%$ higher in the fluticasone/salmeterol treatment group, giving a rate ratio of 1.73 and 1.74 , respectively. When comparing fluticasone/salmeterol with budesonide/formoterol, the pneumonia event rate per 100 patient years was 11.0 versus 6.4. There were 97 deaths from pneumonia in the fluticasone/salmeterol group compared with 52 deaths in the budesonide/formoterol group corresponding to a $76 \%$ increase in mortality. Furthermore, there was no dose-response relation with regard to risk of pneumonia with the two treatments indicating that differences were due to the class of inhaled corticosteroid/long acting $\beta 2$ agonist and not the dose.

This study shows that the risk of pneumonia and pneumonia related events are higher with fluticasone/salmeterol than with budesonide/formoterol in patients with COPD. However, it is important to note that the event rate was higher in the fluticasone/salmeterol group to start with and this was not adjusted for in the analysis. Differences may be related to differences in the immunosuppressant potency between budesonide and fluticasone. Long term randomised controlled trials would be beneficial to assess the extent of such intraclass differences put in context with the benefits of such regimens in preventing exacerbations.

\section{Avais Jabbar}

Correspondence to Dr Avais Jabbar, Department of General Medicine, Northampton General Hospital NHS Trust, Northampton NN1 5BD, UK; ajabbar1@nhs.net

Competing interests None.

Provenance and peer review Not commissioned; internally peer reviewed.

To cite Jabbar A. Thorax 2013;68:996.

Published Online First 1 August 2013

Thorax 2013;68:996. doi:10.1136/thoraxjnl-2013-204098

\section{REFERENCE}

1 Janson C, Larsson $\mathrm{K}$, Lisspers $\mathrm{KH}$, et al. Pneumonia and pneumonia related mortality in patients with COPD treated with fixed combinations of inhaled corticosteroid and long acting $\beta 2$ agonist: observational matched cohort study (PATHOS). BMJ 2013;346:f3306. 